\title{
Spatiotemporal Analysis of Local Field Potentials and Unit Discharges in Cat Cerebral Cortex during Natural Wake and Sleep States
}

\author{
Alain Destexhe, Diego Contreras, and Mircea Steriade \\ Laboratoire de Neurophysiologie, Faculté de Médecine, Université Laval, Québec G1K 7P4, Canada
}

The electroencephalogram displays various oscillation patterns during wake and sleep states, but their spatiotemporal distribution is not completely known. Local field potentials (LFPs) and multiunits were recorded simultaneously in the cerebral cortex (areas 5-7) of naturally sleeping and awake cats. Slowwave sleep (SWS) was characterized by oscillations in the slow $(<1 \mathrm{~Hz})$ and delta $(1-4 \mathrm{~Hz})$ frequency range. The high-amplitude slow-wave complexes consisted in a positivity of depth LFP, associated with neuronal silence, followed by a sharp LFP negativity, correlated with an increase of firing. This pattern was of remarkable spatiotemporal coherence, because silences and increased firing occurred simultaneously in units recorded within a $7 \mathrm{~mm}$ distance in the cortex. During wake and rapideye-movement (REM) sleep, single units fired tonically, whereas LFPs displayed low-amplitude fast activities with increased power in fast frequencies $(15-75 \mathrm{~Hz})$. In contrast with the widespread synchronization during SWS, fast oscillations dur- ing REM and wake periods were synchronized only within neighboring electrodes and small time windows (100-500 $\mathrm{msec}$ ). This local synchrony occurred in an apparent irregular manner, both spatially and temporally. Brief periods ( $<1 \mathrm{sec})$ of fast oscillations were also present during SWS in between slow-wave complexes. During these brief periods, the spatial and temporal coherence, as well as the relation between units and LFPs, was identical to that of fast oscillations of wake or REM sleep. These results show that natural SWS in cats is characterized by slow-wave complexes, synchronized over large cortical territories, interleaved with brief periods of fast oscillations, characterized by local synchrony, and of characteristics similar to that of the sustained fast oscillations of activated states.

Key words: fast oscillations; slow oscillations; arousal; sleep; spatiotemporal properties; cerebral cortex; coherence
The slow $(<1 \mathrm{~Hz})$ and delta $(1-4 \mathrm{~Hz})$ oscillations appear in the electroencephalogram (EEG) and local field potentials (LFPs) during slow-wave sleep (SWS). The distinction between these two sleep rhythms was demonstrated in intracellular studies from animals showing that the slow oscillation groups delta waves (Steriade et al., 1993a,b) and in EEG recordings showing different dynamics of the two oscillations in humans (Achermann and Borbély, 1997). Under ketamine-xylazine anesthesia, the depthnegative (surface-positive) EEG components of slow oscillations are associated with cellular depolarization, whereas depthpositive (surface-negative) components are associated with cellular hyperpolarization (Steriade et al., 1994; Contreras and Steriade, 1995). In natural SWS, extracellularly recorded neurons fire in coincidence with the depth-negative component of slowwave complexes, whereas the depth-positive component is associated with neuronal silence (Steriade et al., 1996; Amzica and Steriade, 1997). Similar relations were found for delta waves in the intact brain (Buzsáki et al., 1988) or isolated cortex (Frost et al., 1966). Thus it seems that slow-wave complexes are characterized by alternating periods of neuronal silence and periods of increased firing. These modulations of firing rate are also in

\footnotetext{
Received Dec. 7, 1998; revised Feb. 12, 1999; accepted March 10, 1999.

This research was supported by the Medical Research Council of Canada and the Human Frontier Science Program.

Correspondence should be addressed to Dr. A. Destexhe, Laboratoire de Neurophysiologie, Faculté de Médecine, Université Laval, Québec G1K 7P4, Canada.

Dr. Contreras's present address: Department of Neuroscience, University of Pennsylvania, School of Medicine, Philadelphia, PA 19104.

Copyright (C) 1999 Society for Neuroscience 0270-6474/99/194595-14\$05.00/0
}

agreement with analyses of the temporal patterns of discharge in the neocortex, which reported that bursts of action potentials and long periods of silence appear exclusively during SWS (Hubel, 1959; Evarts, 1964; Steriade et al., 1974).

By contrast, during aroused states, cortical cells fire tonically (Hubel, 1959; Evarts, 1964; Steriade et al., 1974), and the EEG is dominated by low-amplitude fast activity in the $\beta-\gamma(15-75 \mathrm{~Hz})$ frequency range. Experimentally, fast oscillations can be evoked in awake animals using stimuli requiring attentive behavior (Lopes da Silva et al., 1970; Freeman and Van Dijk, 1987; Rougeul et al., 1979; Bouyer et al., 1981; Murthy and Fetz, 1992), in response to visual stimuli in cats (Eckhorn et al., 1988; Gray et al., 1989), as well as during auditory responses in humans (Ribary et al., 1991). They also occur spontaneously during rapid-eyemovement (REM) sleep in humans (Llinás and Ribary, 1993). The relationship of fast oscillations with unit discharge consistently showed that unit discharges are correlated with negative deflections in the depth EEG (Eckhorn et al., 1988; Gray and Singer, 1989; Murthy and Fetz, 1992; Steriade et al., 1996).

In anesthetized preparations, slow-frequency oscillations are characterized by large-scale coherence, whereas fast oscillations display more local coherence (Bullock and McClune, 1989; Steriade and Amzica, 1996). Fast oscillations are synchronized locally in both space and time, as shown by the very restricted cortical areas and time windows in which coherent fast oscillations appear (Eckhorn et al., 1988; Gray et al., 1989; Steriade and Amzica, 1996). It is however not known whether the same conclusions also apply to oscillations occurring in waking or naturally sleeping animals. 
In this paper, we have investigated the spatiotemporal patterns of oscillations in cat suprasylvian cortex during waking and natural sleep. The field potentials and the discharge of units were compared, as a function of time, distance, and behavioral state of the animal.

Parts of this paper have been published previously (Destexhe et al., 1998b).

\section{MATERIALS AND METHODS}

Multisite field potentials and unit discharges were recorded from the cerebral cortex of cats and subsequently analyzed. The experimental procedures and methods for data analysis are described successively.

\section{Experimental procedures}

Preparation, anesthetics, and chronic implantation. Four adult cats weighting 2.5-3.5 kg were used in the present study. Chronic implantation of recording and stimulating electrodes was performed under ketamine (15 $\mathrm{mg} / \mathrm{kg}$, i.m.), followed by pentobarbital ( $25 \mathrm{mg} / \mathrm{kg}$, i.p.). Cats were implanted in a stereotaxic position, and four screws were placed protruding from the dental cement to hold the cat's head rigid without pain or pressure during recording sessions. Two teflon-insulated wires were inserted in the neck muscles to record the electromyogram (EMG), and two silverball electrodes were cemented into the supraorbital cavity for recording the electrooculogram (EOG). Stainless steel screws were anchored to the bone overlaying the pericruciate and the suprasylvian cortex of the right hemisphere to monitor the gross EEG. The bone overlaying the left suprasylvian cortex was removed, and the exposed dura was covered with a plate of acrylic with holes for the passage of the recording and stimulating electrodes. Buprenorphine $(0.03 \mathrm{mg} / \mathrm{kg}, \mathrm{i} . \mathrm{m}$.) was given every $12 \mathrm{hr}$ during the first 2 postoperative days to prevent pain after surgery. The antibiotic bicillin was injected intramuscularly for $3 \mathrm{~d}$ after surgery. Recordings started 1 week after implantation. Animals slept ad libitum between experimental sessions.

Recording and stimulation. All recordings reported here were obtained from cat suprasylvian gyrus (areas 5-7) by means of stainless steel bipolar coaxial electrodes $(0.1 \mathrm{~mm}$ in diameter $)$ inserted perpendicularly to the cortical surface, with the ring placed on the pia and the tip inserted at a depth of $0.8-1.0 \mathrm{~mm}$. In some cases, extracellular units and LFPs were recorded using monopolar tungsten electrodes with tip resistances from 1 to $5 \mathrm{M} \Omega$. Both coaxial and tungsten electrodes were aligned along the anteroposterior axis of the gyrus in arrays of eight equally spaced electrodes with an interelectrode distance of $1 \mathrm{~mm}$ (Fig. 1, top). The electrodes could be moved independently and were lowered through the holes of an acrylic plate. For bipolar recordings, the polarity was adjusted such that the sharp potentials of slow-wave complexes are negative. For monopolar recordings, the reference electrode was placed in the neck muscles.

Data acquisition. Signals were recorded on an eight-channel digital recorder (Instrutech, Mineola, NY) with an internal sampling rate of $11.8 \mathrm{kHz}$ per channel and four-pole Bessel filters. For LFPs, data were digitized off-line at $250 \mathrm{~Hz}$ using the Igor software package (Wavemetrics; analog-to-digital board from GW Instruments; low-pass filter of 100 $\mathrm{Hz}$ ). Units were digitized off-line at $10 \mathrm{kHz}$, and spike sorting and discrimination were performed with the DataWave software package (DataWave Technologies; filters were $300 \mathrm{~Hz}$ high-pass and $5 \mathrm{kHz}$ low-pass). Data were transferred to a Unix workstation for analysis (see below).

\section{Data analysis}

Data analyses were based on LFPs recorded at equidistant sites and represented by:

$$
v\left(r_{1}, t\right) v\left(r_{2}, t\right) \ldots v\left(r_{n,}, t\right)
$$

where $r_{1} \ldots r_{n}$ are $n$ equidistant cortical sites and obey the relation $r_{i+1}$ $=r_{i}+\Delta r$, where $\Delta r$ is the interelectrode distance (1 $\mathrm{mm}$ in this case). LFPs were normalized by subtraction of their average value and division by their SD.
Temporal correlations. Temporal correlations, or cross-correlations, are linear estimators to measure temporal variations of coherence of a signal. They were computed according to the relation:

$$
C_{i j}(\tau)=\frac{\sum_{k} v\left(r_{i}, t_{k}\right) v\left(r_{j}, t_{k}+\tau\right)}{\sum_{k} v\left(r_{i}, t_{k}\right)^{2}},
$$

where the correlation expresses the average of the normalized LFP $v\left(r_{i}, t_{j}\right)$ at site $r_{i}$ and time $t_{j}$, multiplied by the normalized LFP $v\left(r_{j}, t_{k}+\tau\right)$ at site $r_{j}$ and time $t_{k}+\tau$. $C_{i j}(\tau)$ varies between -1 and +1 . Efficient algorithms based on fast Fourier transforms were used to evaluate $C_{i j}(\tau)$ (Press et al., 1986).

The autocorrelation $C_{i i}(\tau)$ is obtained by setting $i=j . C_{i i}(\tau)$ measures how a signal is temporally coherent with itself: its value stays close to unity as long as the signal is correlated; it oscillates for periodic oscillations and decays toward zero for irregular signals.

Spatial correlations. The spatial correlation was computed according to the relation:

$$
C(x)=\frac{\sum_{i, k} v\left(r_{i}, t_{k}\right) v\left(r_{i}+x, t_{k}\right)}{\sum_{i, k} v\left(r_{i}, t_{k}\right)^{2}},
$$

where $v\left(r_{i}, t_{k}\right)$ is the normalized LFP at site $r_{i}$ and time $t_{k}$. In this case, the correlation is expressed as the time average of the product of every possible pair of sites, combined for all pairs with the same intersite distance $(x) . C(x)$ is included between -1 and +1 .

This quantity measures the coherence of the spatial portrait of the system: if all variables $v\left(r_{i}, t_{k}\right)$ are irregular in time but coherent in space (similar signals in different sites $r_{i}$ ), then $C(x)$ will be close to unity. This is indeed the case for slow-wave sleep (see Results). Other oscillation types, such as fast oscillations, may show variations of coherence in both time and space (see Results). In this case, it is necessary to use both temporal and spatial correlations to characterize these two aspects independently. A closely related-but different-measure of spatial coherence is seen by representing the peak of the cross-correlation as a function of distance. Using either procedure yielded no appreciable difference for the signals studied here.

Space constant. The representation of $C(r)$ as a function of distance $r$ was used to measure the decay of correlation with distance in the anteroposterior axis of the suprasylvian cortex. Because the decay of spatial correlations was always smooth toward zero, a first-order decaying exponential term was used to fit the correlation data:

$$
C(x)=\exp (-x / \lambda)
$$

where the correlation space constant $\lambda$ is a measure of the spatial extent of the coherence of a spatially homogeneous phenomenon, similar to the coherence measure based on power spectra (Bullock and McClune, 1989).

In some cases, a biexponential fit was performed:

$$
C(x)=A \exp \left(-x / \lambda_{1}\right)+(1-A) \exp \left(-x / \lambda_{2}\right),
$$

where $\lambda_{1}$ and $\lambda_{2}$ are two space constants and $A$ is a constant factor determining the relative amplitude of the two exponentials.

Time-dependent correlations. In some cases, it was necessary to display temporal variations of coherence. In this case, the time was sliced into consecutive time windows of fixed duration, and $C_{i j}(\tau)$ or $C(r)$ was computed within each window (all signals were renormalized in each window). The values of correlations were then represented as a function of the time value corresponding to the beginning of each window.

Spatiotemporal maps. A useful representation of multisite signals is a spatiotemporal map consisting of successive snapshots of the distribution of electrical activity across the cortex. Snapshots were generated by assigning a gray spot to the instantaneous value of LFP at each electrode and arranging spots along a vertical line (anteroposterior axis is bottomto-top). Successive snapshots were then arranged horizontally in columns, defining a map in which LFP is represented as a function of space and time. This map offers a compact representation of the spatiotemporal dynamics of the system, in which synchronous events appear as vertical straight lines and oblique lines stand for propagating waves or phase shifts.

Time-dependent power spectra. In addition to displaying variations of spatial and temporal coherence, the signals also displayed significant variations of oscillation frequency. To characterize this aspect, we evaluated power spectra in successive time windows, similar to correlations. 

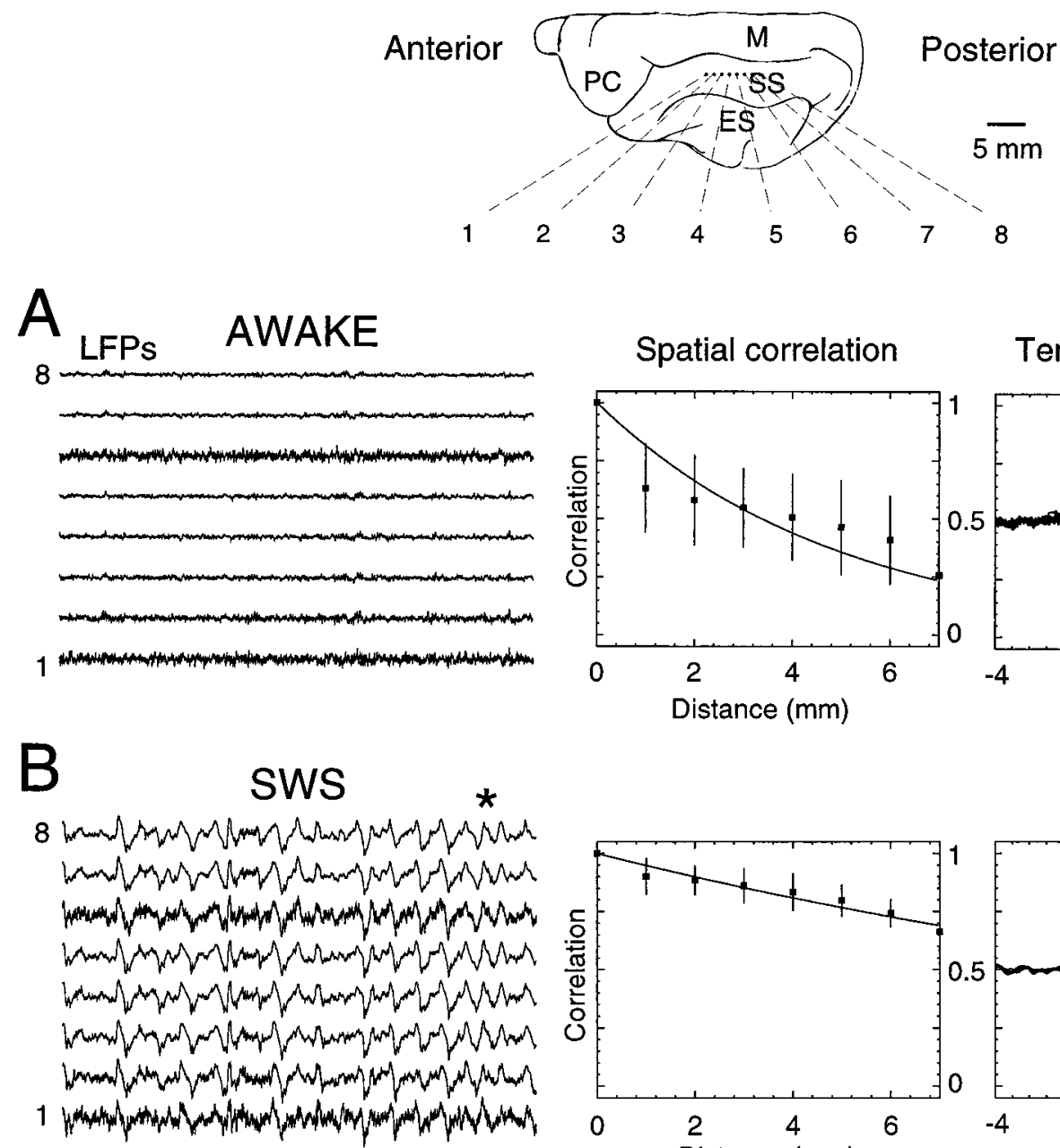

Temporal correlation

B

Distance $(\mathrm{mm})$
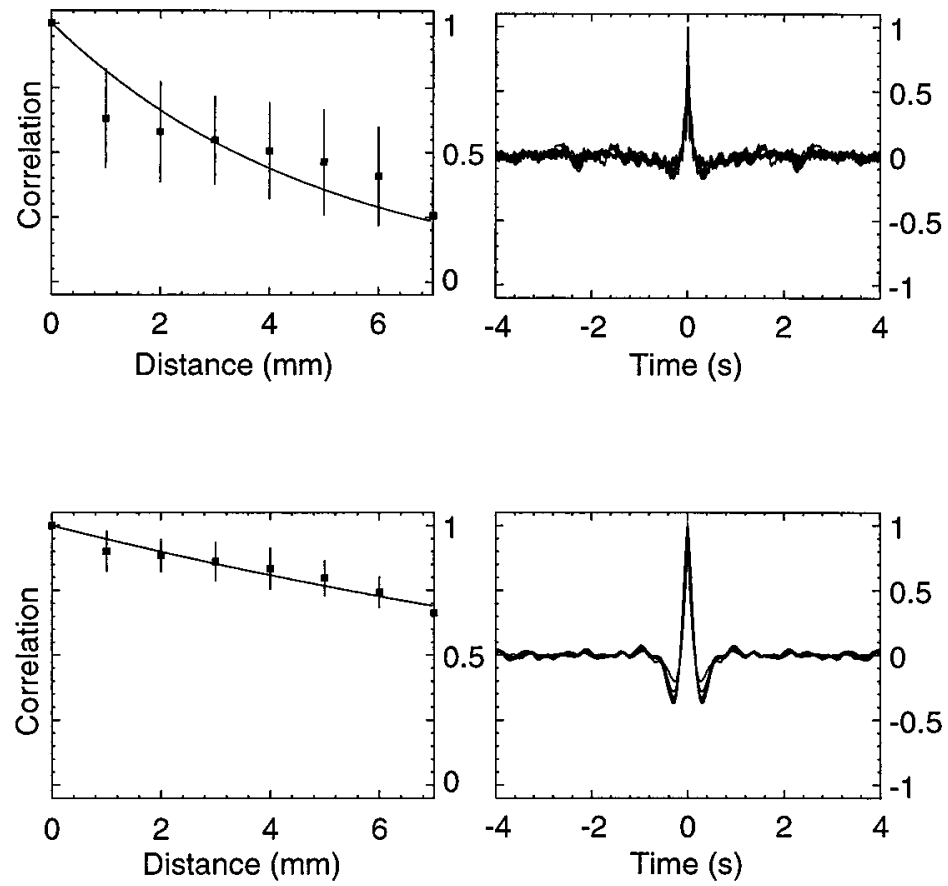

C

REM
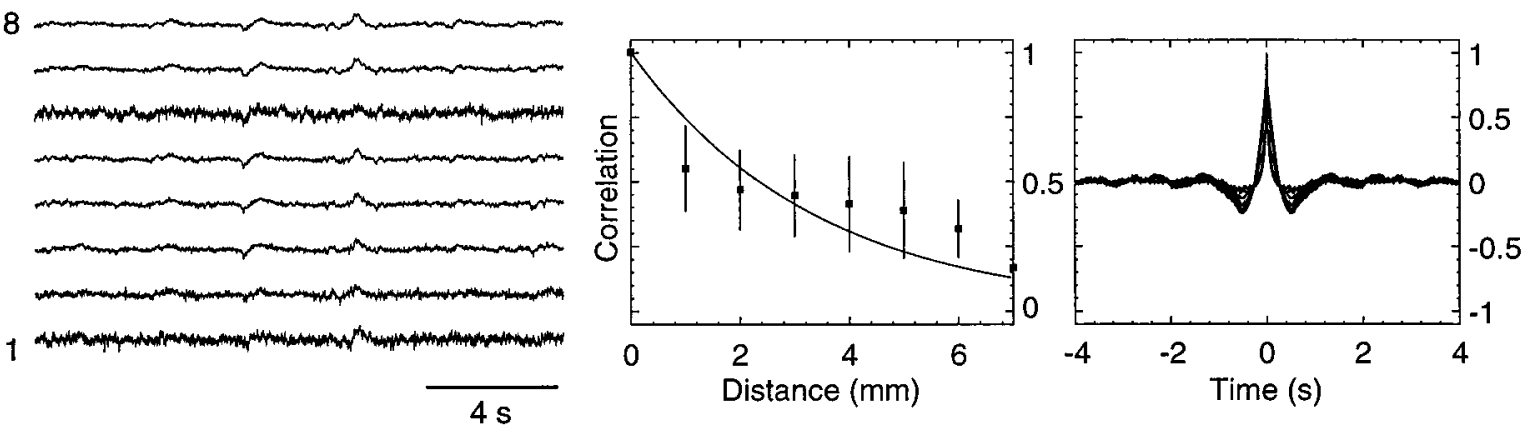

Figure 1. Multisite local field potentials in cat cerebral cortex during natural wake and sleep states. Top, Eight bipolar electrodes (interelectrode distance of $1 \mathrm{~mm}$ ) were inserted into the depth $(1 \mathrm{~mm})$ of areas 5-7 of cat neocortex. $E S$, Ectosylvian gyrus; $M$, marginal gyrus; $P C$, postcruciate gyrus; $S S$, suprasylvian gyrus. LFPs (LFPs), the decay of correlation with distance (Spatial correlation), and autocorrelations (Temporal correlation) are shown for three different states. $A$, During the wake state $(A W A K E)$, LFPs were characterized by low-amplitude fast activities in the $\beta-\gamma$ frequency range (15-75 $\mathrm{Hz}$ ). Correlations decayed steeply with distance and time. $B$, During SWS, the LFPs were dominated by large-amplitude slow-wave complexes recurring at a slow frequency $(<1 \mathrm{~Hz})$ and displaying high coherence. Slow-wave complexes of higher frequency $(1-2 \mathrm{~Hz})$ were also present and displayed more moderate coherence (asterisk). Correlations stayed high for large distances (Spatial correlation) but decayed steeply with time (Temporal correlation). C, During REM sleep, LFPs and correlations had characteristics similar to those during wake periods.

The power spectrum was evaluated using fast Fourier transforms (see Press et al., 1986), and the relative power in a given frequency band (power within that frequency band divided by the total power) was represented as a function of time. Fourier transforms were also used for digital filtering, using either square or Hamming windows (Press et al., 1986), with no measurable effect on the present results.
Wave-triggered averages. Wave-triggered averages consist in averaging short windows of data selected by reference to the time of occurrence of a given trigger waveform. Negative peaks of LFPs were used as the trigger and were detected numerically using a thresholding procedure. The peak was calculated such that only one peak was present within an interval of the same order as the oscillation cycle $(200-1000 \mathrm{msec}$ for 

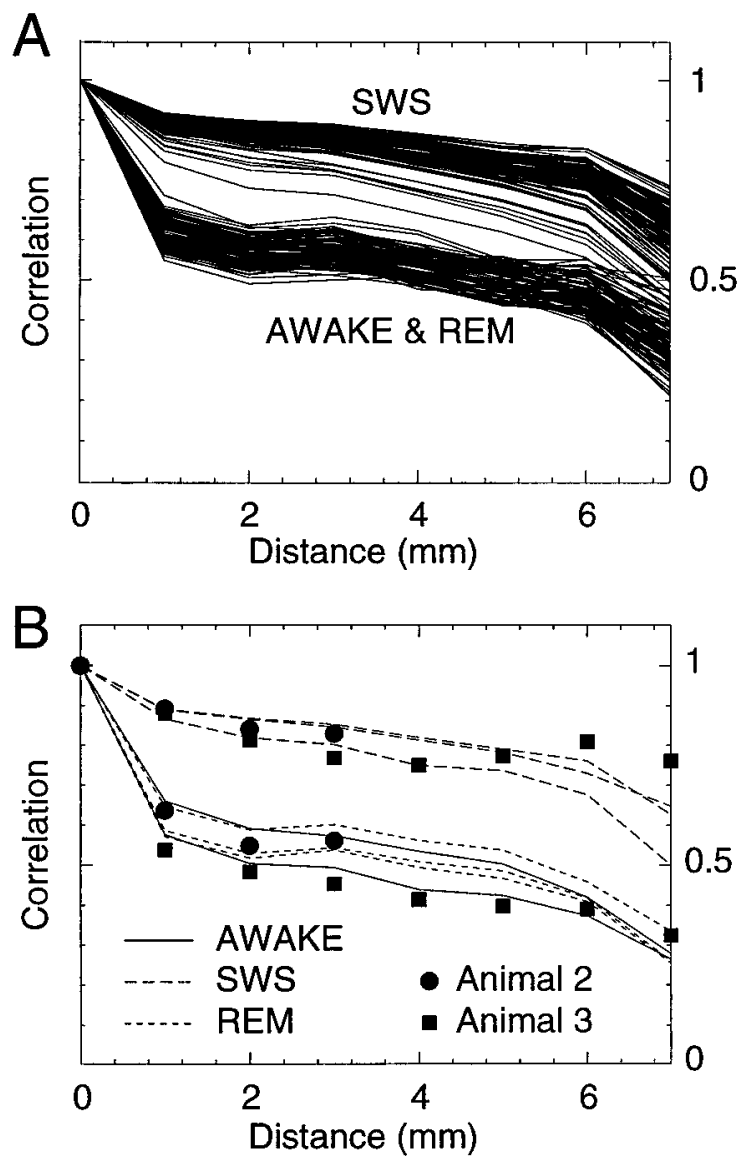

Figure 2. Spatial correlations during wake and sleep states. $A$, Stationarity of spatial correlations in a $2 \mathrm{hr}$ recording in the same animal. Each solid line represents the correlations calculated in consecutive windows of $20 \mathrm{sec}$ in a total recording time of $2 \mathrm{hr}$. Wake and REM periods were indistinguishable, but SWS displayed significantly higher correlations. $B$, Spatial correlations calculated from long periods of time in different animals. Lines indicate several periods of wake, SWS, and REM sleep in the same animal (length of each period, 2.5 and 15 min for wake state; 11 , 15, and $17 \mathrm{~min}$ for SWS; and 3, 8, and $10 \mathrm{~min}$ for REM). The symbols indicate the spatial correlations obtained for two other animals (squares, $5 \mathrm{~min}$ of SWS and $4 \mathrm{~min}$ of wake; circles, $8 \mathrm{~min}$ of SWS and $4 \mathrm{~min}$ of REM). All data sets were obtained using the eight-electrode setup shown in the top of Figure 1, except for the data set shown by circles that was obtained with four electrodes (interelectrode distance of $1 \mathrm{~mm}$ in all cases).

SWS; $10-40 \mathrm{msec}$ for fast oscillations). The signals in a given time window around the peak were then retained for averaging.

In some cases, multiunit discharges were averaged by reference to negative peaks of the LFPs. This type of wave-triggered average estimates the average pattern of firing correlated with the LFP negativity. To estimate the significance of these estimations, we always compared wave-triggered averages with a control value. The control consisted in repeating the same procedure using randomly shuffled spikes, in which the same number of spikes was distributed randomly across the time axis. This control eliminates spurious estimates attributable to poor statistics or artifacts.

Spike-triggered averages. To detect correlations between spikes and LFPs, a similar averaging procedure was performed in which spike events were used as the reference time to select the windows for averaging. In this case, spike-triggered averages estimate the LFP waveform preferentially associated to spike events. Because spikes are usually highly variable, it is necessary to use large number of spikes to yield statistically significant results. In this paper, all spike-triggered averages were compared with a control analysis based on randomly shuffled spikes (see above), thus providing an estimate of the statistical significance of the results.
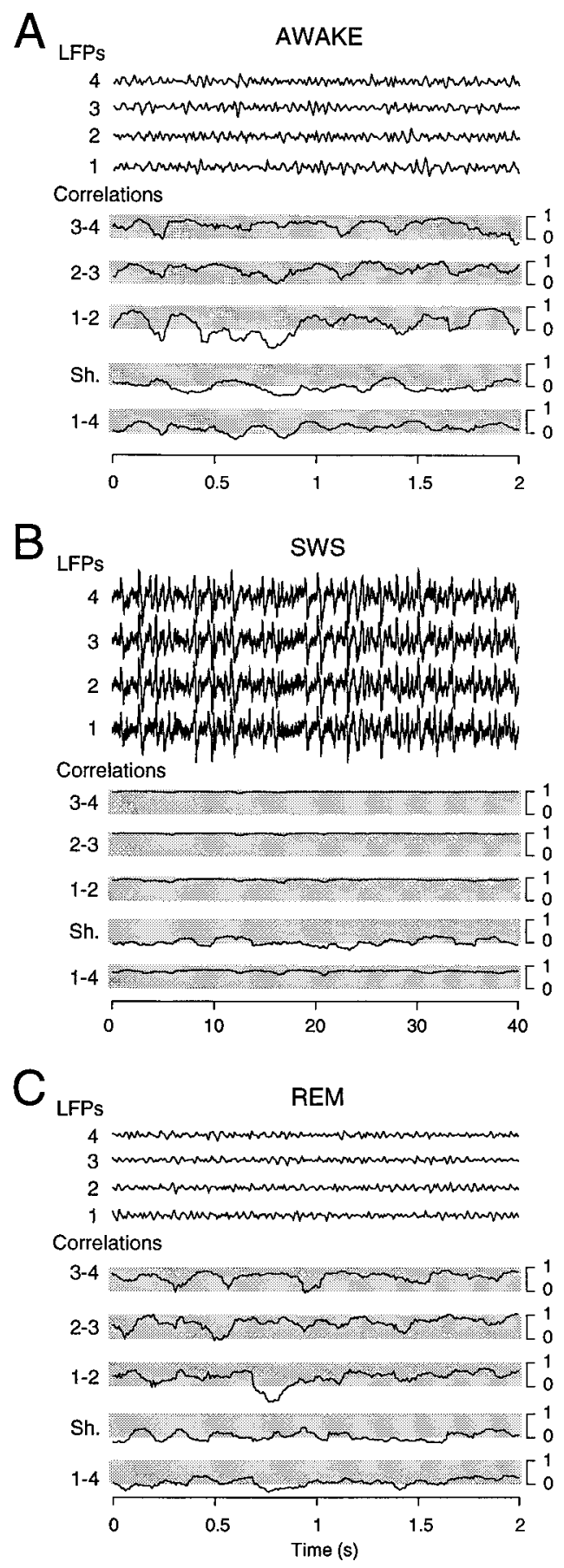

Figure 3. Fast oscillations are coherent locally in space and time. LFP recordings in the suprasylvian gyrus ( $L F P$; locations similar to that of electrodes 1-4 in Fig. 1, with a $1 \mathrm{~mm}$ interelectrode distance) are shown together with the maximal cross-correlation (Correlations) calculated between pairs of electrodes (1-2, 2-3,3-4, and 1-4 pairs) Sh., The control correlation obtained between electrode 1 and the same signal taken $20 \mathrm{sec}$ later. $A$, Fast oscillations during wake periods. Neighboring electrodes were occasionally synchronized, as shown by correlations close to 1 , but only for short periods of time $(100-500 \mathrm{msec})$. $B$, Period of slow-wave sleep with the number of oscillation cycles similar to that in $A$ (note the difference in the time scale). In this case, correlations between neighboring electrodes stayed close to unity, and the synchrony extended the entire recorded area. $C$, Period of REM sleep. Fast oscillations had a dynamics similar to that in $A$, consisting in brief periods of synchrony between neighboring electrodes, occurring irregularly and within short time windows. Correlations were calculated in successive time windows of 100 msec for $A$ and $C$ and $2 \mathrm{sec}$ for $B$. 


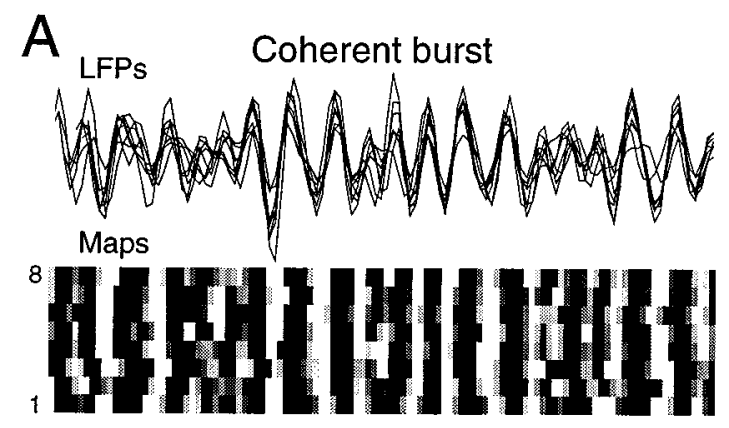

B
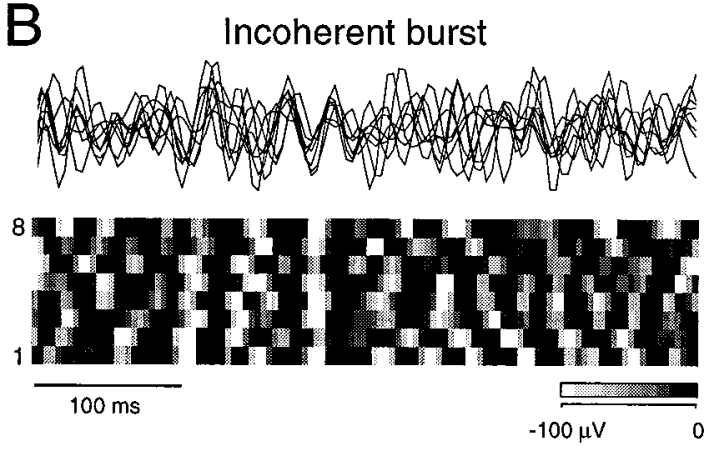
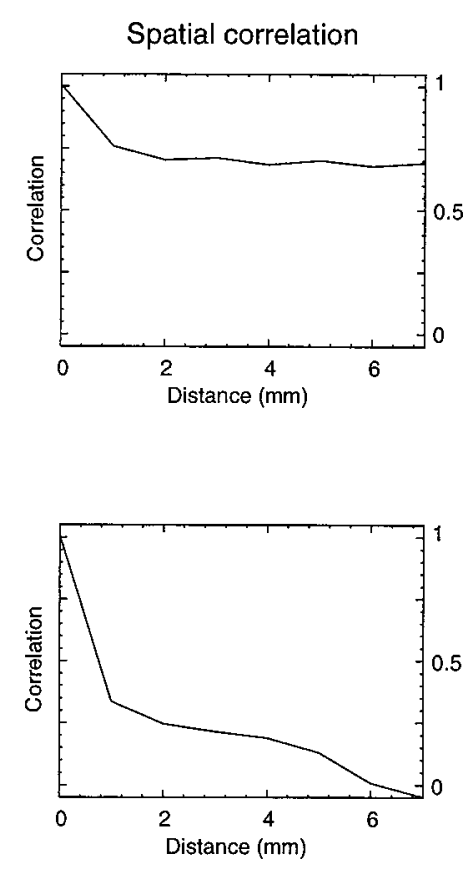

Figure 4. Fast oscillations are occasionally coherent across large cortical distances. LFPs from eight recording electrodes are shown during fast oscillations ( $L F P s$; signal filtered between 15 and $75 \mathrm{~Hz}$ ). Spatiotemporal maps for the same period of activity are shown below the recordings (Maps). Spatiotemporal maps were constructed by representing space ( $y$-axis) and voltage (gray level) against time ( $x$-axis). The gray scale ranged from white $(-100 \mu \mathrm{V}$ and below) to black ( $0 \mu \mathrm{V}$ and above) in 10 levels. The correlation decay with distance calculated during the same period of time is shown on the right (Spatial correlation). A, Coherent burst, Fast oscillations were occasionally synchronized across large distances, as shown by the vertical black-white stripes in the spatiotemporal maps and the high values of correlations at a $7 \mathrm{~mm}$ distance. This coherent burst was recorded during REM sleep. B, Incoherent burst, In most instances, synchrony was present only between neighboring electrodes and during restricted time windows (as shown in Fig. 3). This local synchrony of fast oscillations can also be seen by the local patterns of black-white stripes in the spatiotemporal maps. In this case, correlations decayed steeply with distance. The latter type of activity represents the pattern observed most frequently during wake and REM periods.
All analyses were performed using C programs based on a library of numerical algorithms (Press et al., 1986) and were run on a Sparc 20 workstation (Sun Microsystems, Mountain View, CA).

\section{RESULTS}

Two oscillation types were considered in this study. "Slow oscillations" or "slow-wave complexes" refer to oscillations in the slow $(<1 \mathrm{~Hz})$ and delta $(1-4 \mathrm{~Hz})$ frequency range, whereas "fast oscillations" refer to oscillations in the $\beta-\gamma(15-75 \mathrm{~Hz})$ frequency range. We first describe the spatiotemporal properties of these two oscillation types in awake, SWS, and REM sleep. We then investigate the relation between unit firing and field potentials for each type of oscillation. Finally, brief periods of fast oscillations during slow-wave sleep are analyzed using the same methods.

\section{Spatial and temporal correlations during natural wake and sleep states}

Multisite LFPs were recorded using a set of eight equidistant bipolar electrodes (interelectrode distance of $1 \mathrm{~mm}$; see Fig. 1, top). Wake and sleep states were identified using the following criteria: for wake, low-amplitude fast activity in LFPs and high EOG and high EMG activity; for SWS, LFPs dominated by high-amplitude slow-waves and low EOG and EMG activity present; for REM sleep, low-amplitude fast LFP activity, high EOG activity, and abolition of EMG activity. During waking and attentive behavior, LFPs were characterized by low-amplitude fast $(15-75 \mathrm{~Hz})$ activity (Fig. $1 \mathrm{~A}$, left). During SWS, LFPs were dominated by high-amplitude slow-wave complexes occurring at a frequency of $<1 \mathrm{~Hz}$ (Fig. 1B, left). Slow-wave complexes of higher frequency (1-4 Hz; Fig. 1B, asterisk) and spindle waves (7-14 Hz; data not shown) were also present in SWS. During periods of REM sleep, the activity was similar to that of waking periods (Fig. $1 C$, left).

Autocorrelations evaluated over long periods $(20 \mathrm{sec})$ for each state of the animal showed a relative steep decay toward zero (Fig. 1, right), indicating that the LFP activity is very irregular despite the dominant frequencies characteristic to each state.
This is contrary to the pronounced rhythmicity that can appear in autocorrelations calculated for small time windows (data not shown). For long periods of time, the autocorrelations showed similar behavior for SWS, REM, and waking states and, therefore, cannot be used to distinguish these states.

By contrast, correlations represented as a function of distance displayed marked differences between awake, REM, and SWS (Fig. 1, middle). SWS displays slow-wave complexes of a remarkable spatiotemporal coherence, as indicated by the high values of spatial correlations for large distances, in contrast with the steeper decline of spatial correlations with distance during waking and REM sleep. Spatial correlations were evaluated in different animals and during different wake and sleep episodes in the same animals, and the data grouped together are shown in Figure 2. Correlations calculated in consecutive windows of $20 \mathrm{sec}$ in a total recording time of $2 \mathrm{hr}$ (in the same animal) are shown in Figure $2 A$. There was a clear clustering of curves corresponding to SWS and that obtained during awake and REM, indicating that the differences in spatial correlation shown in Figure 1 are robust. Figure $2 B$ shows spatial correlations evaluated for long periods of time, from 2.5 to $17 \mathrm{~min}$ in different animals. Here again, there was a clear clustering of the spatial correlations according to different wake and sleep episodes.

The evaluation of the correlation space constant for three animals (see Materials and Methods) led to values of $\lambda=3.7 \pm$ $0.4 \mathrm{~mm}$ (mean $\pm \mathrm{SD})$ for waking periods $(n=8), \lambda=13.0 \pm 1.2$ $\mathrm{mm}$ for SWS $(n=15)$, and $\lambda=3.1 \pm 0.5 \mathrm{~mm}$ for REM sleep $(n=$ 11). These values show that the characteristic correlation space constant is much larger in SWS compared with that during fast oscillations. A biexponential fitting (see Materials and Methods) provided better fits for waking periods $\left(\lambda_{1}=10.6 \pm 0.6 \mathrm{~mm} ; \lambda_{2}\right.$ $=0.03 \pm 0.01 \mathrm{~mm})$ and for REM sleep $\left(\lambda_{1}=7.5 \pm 0.5 \mathrm{~mm} ; \lambda_{2}=\right.$ $0.03 \pm 0.01 \mathrm{~mm})$ but not during SWS sleep $\left(\lambda_{1}=16.1 \pm 1.5 \mathrm{~mm}\right.$; $\left.\lambda_{2}=13.3 \pm 0.9 \mathrm{~mm}\right)$. The small value of $\lambda_{2}$ during fast oscillations 

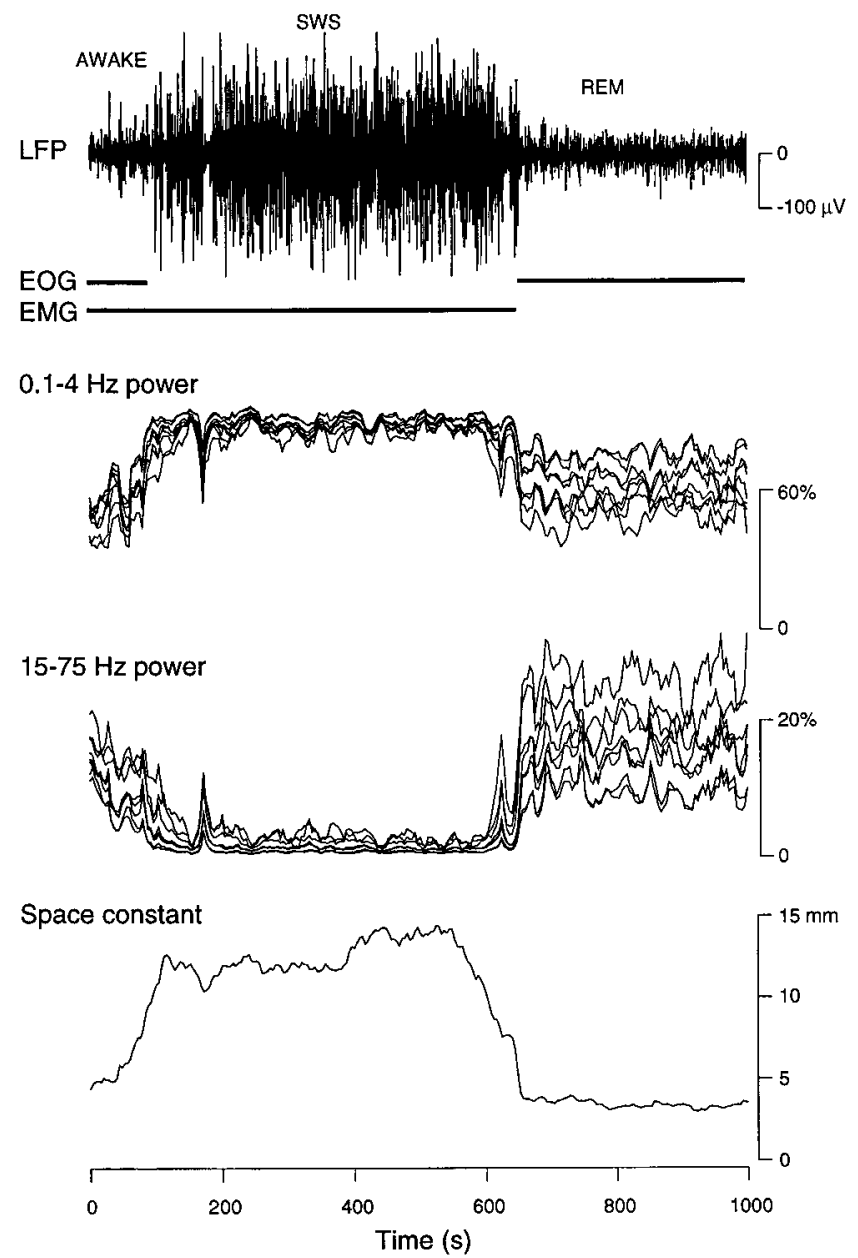

Figure 5. Spatial coherence during the transition between wake and sleep states. Top, The depth LFP recorded in the suprasylvian gyrus during a period of $16 \mathrm{~min}$, consisting of $\sim 2 \mathrm{~min}$ of wake followed by $\sim 8$ min of SWS and $\sim 6$ min of REM sleep, is shown. The presence of ocular movements (EOG) and of muscular tonus (EMG) were monitored and are indicated by horizontal bars. Middle, The relative power of $0.1-4$ and $15-75 \mathrm{~Hz}$ frequency bands are represented during the same period at eight different cortical sites (as shown in the top of Fig. 1). Bottom, The space constant of the decay of correlations with distance is shown. SWS activity is characterized by a marked increase of spatial coherence compared with that of wake and REM periods. Power spectra and spatial correlations were calculated in successive windows of $16.4 \mathrm{sec}$ (4096 points).

betrays a sharp initial drop of correlations, which is followed by a more progressive decay similar to that of SWS.

This analysis shows that SWS, awake, and REM states are distinguished by the behavior of correlations with distance. SWS is spatially coherent and is characterized by high values of correlations across cortical distances of several millimeters. Wake and REM sleep show less spatial coherence, with correlations decaying steeply with distance.

\section{Local coherence of fast oscillations}

The more local coherence of fast oscillations contrasted with the large-scale synchrony of slow waves. This phenomenon was investigated in more detail by monitoring the evolution of local correlations as a function of time. The maximum of the peak of the cross-correlation between two neighboring sites was evaluated within successive time windows of $100 \mathrm{msec}$ duration (2 sec for
SWS to have a similar number of oscillation cycles). The representation of the maximal correlation as a function of time is shown in Figure 3. During waking periods (Fig. $3 A$ ), neighboring electrodes were occasionally synchronized, as shown by correlations close to 1 , but only for short periods of time (100-500 msec). On the other hand, distant electrodes displayed lower correlation values (Fig. 3A, pair 1-4), as did the "shuffled" signal (Fig. 3A, Sh.).

Local correlations had different properties during SWS (Fig. $3 B$ ); correlations between neighboring electrodes tended to stay close to 1 , whereas distant electrodes displayed lower, although still high, correlations (Fig. 3B, pair 1-4). These features are in agreement with the high values of spatial correlations evidenced above. During REM sleep (Fig. 3C), fast oscillations displayed locally correlated dynamics similar to that in the awake animal.

These results indicate that fast oscillations are characterized by brief periods of synchrony between neighboring electrodes, occurring irregularly and within short time windows, by contrast to SWS in which slow-wave complexes always appear coherently over large distances. Occasionally, bursts of fast oscillations were coherent across large distances (Fig. 4A). This synchrony was also apparent in spatiotemporal maps of activity and in the high value of correlations at a $7 \mathrm{~mm}$ distance (Fig. 4A). However, these events were only occasionally seen during REM sleep periods, and the most typical portrait of fast oscillations is rather incoherent spatiotemporally (Fig. 4B), with significant correlations only appearing between neighboring sites and within restricted time windows.

These results show that fast oscillations are characterized by local synchrony but may be occasionally coherent over large distances. Fast oscillations seem therefore to be characterized by a succession of coherent events occurring locally both in space and time.

\section{Spatial coherence during the transition between wake and sleep states}

The classical electrographic criteria to assess the transition between wake and sleep states are the patterns of electrical activity (as recorded by EEGs or LFPs), the level of muscular tone (as recorded by the EMG), and the presence of ocular movements (detected by the EOG). Here we show that the spatial coherence varies in accordance with these classic criteria.

The time course of LFPs, EOGs, and EMGs are shown during the transition between wake and sleep states in Figure 5. Waking periods were characterized by muscular tonus, ocular movements, and low-amplitude fast LFP activity (Fig. 5, $A W A K E$ ). SWS was characterized by muscular tonus, no ocular movements, and LFP activity dominated by high-amplitude slow-wave complexes (Fig. $5, S W S$ ). REM sleep was characterized by the absence of muscular tonus, prominent eye movements, and low-amplitude fast activity in LFPs (Fig. 5, REM). In addition to these classic criteria, the spatial coherence was evaluated by the correlation space constant $\lambda$, the robustness of which was shown in Figure 2. During SWS, the correlation space constant was characterized by significantly higher values than that in wake and REM sleep and stayed high for the entire duration of SWS, as detected by LFP, EOG, and EMG.

\section{Slow-wave complexes are preceded by a generalized neuronal silence}

LFPs and units were separated by standard procedures (see Materials and Methods). During the waking state, units tended to 

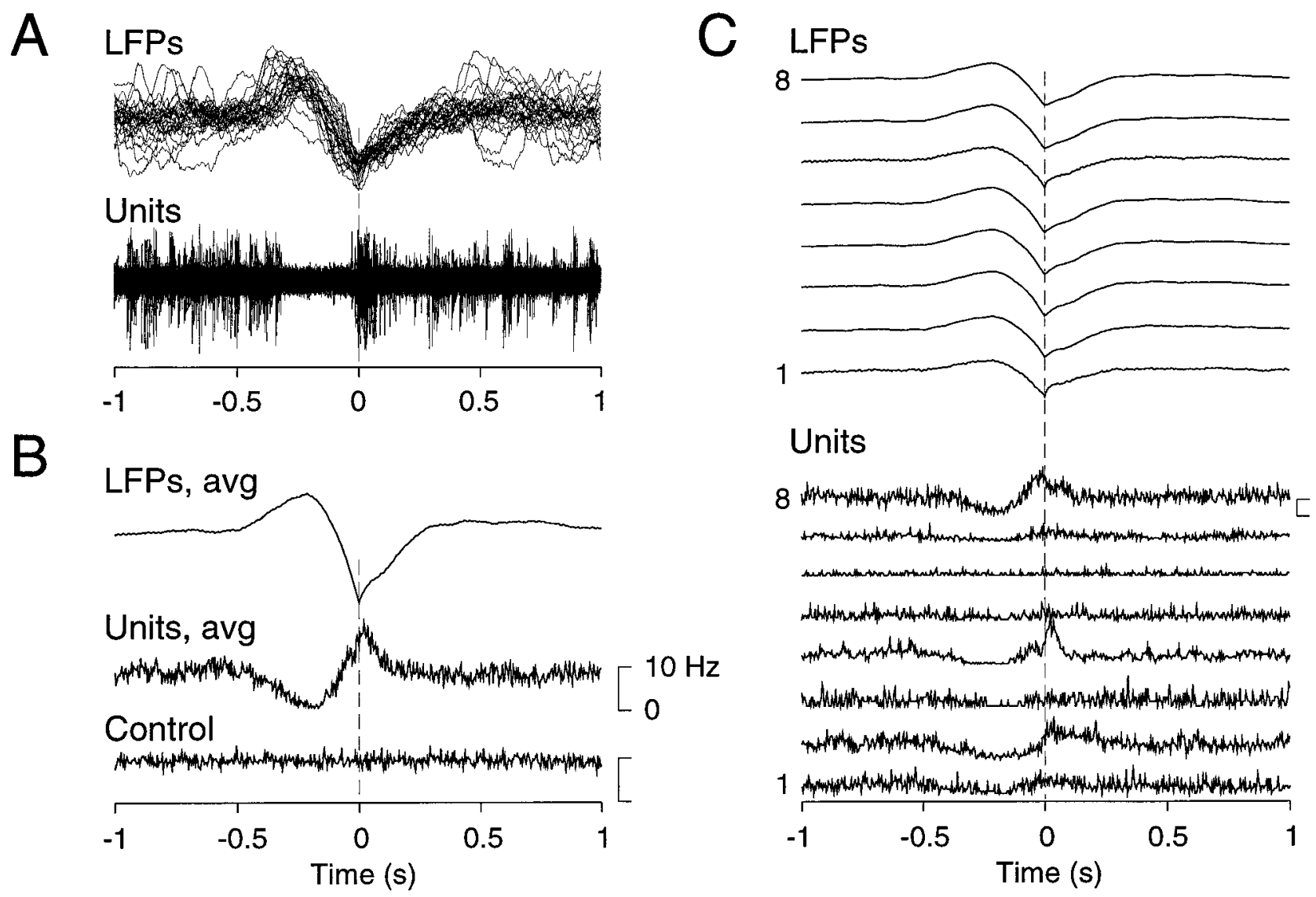

Figure 6. Relation between simultaneously recorded multiunit discharges and field potentials during slow-wave complexes. $A$, Individual slow-wave complexes were detected numerically during SWS and were aligned with respect to the negative peak of the LFPs (LFPs). The multiunit discharges detected in the same electrode were aligned similarly (Units). B, Wave-triggered averages of field potentials and multiunit discharges are shown. The averaged field potentials ( $L F P s, a v g$ ) were constructed by averaging the LFP over the eight electrodes and over 210 detected slow-wave complexes. The resulting averaged LFP consisted in a slow positivity followed by a sharp negativity. The corresponding multiunit discharges were averaged similarly (Units, avg) and displayed a drop of firing rate correlated with LFP positivity, followed by an increase of firing during the LFP negativity. The same wave-triggered average did not show any modulation of firing rate if performed on randomly shuffled spikes $($ Control $)$. $C$, Spatial profile of the relation between units and LFPs is presented. Local field potentials, averaged over 210 slow-wave complexes, are shown for each electrode ( $L F P s)$. The corresponding wave-triggered averages of multiunit discharge at each electrode are shown (Units). Slow-wave complexes consisted in a widespread drop of firing, correlated with LFP positivity, followed by a synchronized increase of firing, correlated with LFP negativity. These events were synchronous over the entire extent of the cortical area recorded $(7 \mathrm{~mm})$. Data in $A-C$ are from the same animal.

discharge tonically, similar to previous observations (Hubel, 1959; Evarts, 1964; Steriade et al., 1974). The relation between units and LFP was not evident at first sight, although there was a tendency to discharge during LFP negativity (see below). During SWS, the pattern of discharge was more phasic and characterized by periods of silences and of increased firing, as reported previously (Evarts, 1964; Steriade et al., 1974). Positive deflections of slow-wave complexes were almost always associated with a neuronal silence in all units, whereas negative deflections tended to be correlated with a brief increase of firing (Fig. 6A). REM sleep displayed activity patterns similar to that in awake animals.

The temporal modulations of unit firing during SWS were investigated by calculating wave-triggered averages. Superposition of LFPs and unit activity showed that slow-wave complexes almost invariably correlate with a silence in the units (Fig. 6A). Wave-triggered averages calculated over 210 complexes revealed a marked modulation of firing rate associated with slow-wave complexes (Fig. 6B, Units, avg), whereas the same procedure performed with randomly shuffled spikes did not show any pattern (Fig. 6B, Control). This analysis demonstrates a drop of firing rate in close correspondence to the depth positivity of slow-wave complexes, whereas depth negativity is associated with an increase of neuronal firing (Fig. 6B), in agreement with previous intracellular observations (Contreras and Steriade, 1995; Steriade et al., 1996).

The same analysis was also performed as a function of distance (Fig. 6C). Wave-triggered averages performed simultaneously between the LFP and cells at each electrode showed that the depth positivity of slow-wave complexes corresponded to a concerted silence in almost all units, whereas the depth negativity was correlated with an increased firing. Only one unit was not correlated with LFP (Fig. 6C, unit 6). In other experiments with less electrodes, all units were correlated with LFP (data not shown). This analysis shows that SWS complexes are characterized by a generalized decrease of firing occurring over large cortical distances, followed by an increased firing occurring in rebound to each period of silence.

\section{Increased firing probability during the negative field potential of fast oscillations}

During wake and REM sleep, units fired tonically, and their relation with LFPs was not evident at first sight. We performed 

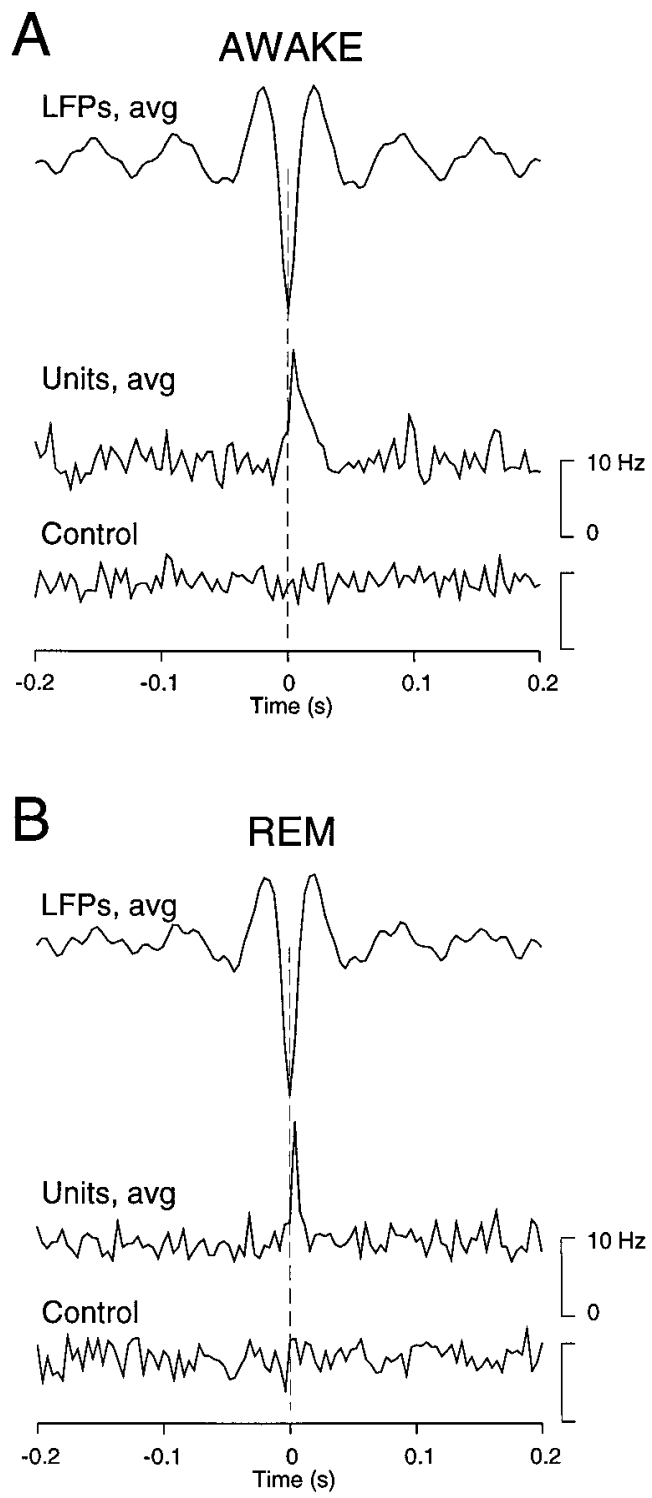

Figure 7. Relation between simultaneously recorded multiunit discharges and field potentials during fast oscillations of wake and REM sleep. $A$, Relation between local field potentials ( $L F P s, a v g$ ) and multiunit discharges (Units, avg) in periods of wake. Signals were filtered between 15 and $75 \mathrm{~Hz}$, and the peak negativities of field potentials were detected. The LFP waveform shown was obtained by averaging over a total of 467 detected events from eight electrodes. The corresponding wave-triggered average of multiunit discharges displayed a marked increase of firing correlated with the LFP negativity. The same analysis performed on randomly shuffled spikes did not show any pattern (Control). B, Same analysis during periods of REM sleep in the same animal. In this analysis, 1721 detected events were used to compute the averaged LFP. During REM sleep, similar to wake states, cells tended to fire in relation with the negativity of the field potentials during fast oscillations, whereas no increase of firing was seen in the control. Data in $A$ and $B$ are from the same animal.

the same wave-triggered average procedures during fast oscillations of waking periods using the peak negativity of LFP to trigger the averaging procedure. Wave-triggered averages computed from a total of 467 events indeed showed that units were significantly correlated with LFPs (Fig. 7A, Units, avg), whereas the same procedure applied to randomly shuffled spikes did not reveal any pattern (Fig. $7 \mathrm{~A}, \mathrm{Control}$ ). This analysis therefore demonstrates a marked increase of firing in correspondence with the depth-negative component of fast oscillations, as shown previously in anesthetized animals (Eckhorn et al., 1988; Gray and Singer, 1989; Murthy and Fetz, 1992; Steriade et al., 1996). The same conclusions were also obtained from fast oscillations of REM sleep (Fig. 7B).

However, the existing correlations between units and LFPs during waking or REM sleep were only seen for signals emanating from the same electrode. Attempts to find correlations between the LFP of one electrode and units recorded by other electrodes, at a distance of $4 \mathrm{~mm}$ and more, were unsuccessful during wake periods (Fig. $8 A$ ). On the other hand, a significant correlation was evident during SWS (Fig. $8 B$ ), in agreement with the large-scale correlations found in Figure 6. During REM sleep, no correlations were present over large distances (Fig. 8C).

The relations between extracellular unit discharges and LFP activity can also be detected from the analysis of spike events. Spike-triggered averages of LFPs computed in waking periods show that spikes occurred preferentially in correspondence with a depth-negative component of the field potential (Fig. 9A, LFPs, $a v g$ ), whereas randomly shuffled spikes yielded a flat line (Fig. $9 A$, Control). A similar relation was obtained during REM sleep (Fig. $9 B$ ). By contrast, performing the same spike-triggered averaging procedure for periods of SWS yielded an average LFP consisting of a broad depth-negative deflection, followed by a slow depthpositive component (Fig. 9C, LFPs, avg), whereas no pattern was obtained using randomly shuffled spikes (Fig. 9C, Control).

This analysis therefore confirms the above conclusions; during wake and REM sleep, cortical neurons tend to fire in correspondence with the depth-negative component of fast oscillations recorded by the same electrode. By contrast, during SWS, neuronal firing is coherent across large distances and consists in periods of decreased and increased firing, correlated with the depthpositive and depth-negative component of slow-wave complexes, respectively.

\section{Periods of fast oscillations occur during slow-wave sleep}

Although SWS is clearly dominated by high-amplitude slow-wave activity, at closer scrutiny, it seems that SWS also contains brief periods of low-amplitude fast oscillations. In LFPs, examining the distribution of dominating frequencies using fine time windows $(\sim 0.5 \mathrm{sec})$ reveals that periods of slow-wave complexes, with high power in low-frequency $(0.1-4 \mathrm{~Hz})$ bands, alternate with periods dominated by frequencies in the $\beta-\gamma$ range $(15-75 \mathrm{~Hz}$ ) (Fig. 10). It thus seems that SWS is composed of slow-wave complexes separated by brief periods of fast oscillations, in agreement with previous observations (Steriade et al., 1996).

Investigating the correlation decay as a function of distance based on multisite LFPs (see above) reveals that periods of slow-wave complexes display high coherence (Fig. 10, single asterisk), whereas the coherence is markedly diminished during brief periods of fast oscillations (Fig. 10, double asterisks). This local analysis of coherence suggests that the brief periods of fast oscillations occurring during SWS have characteristics similar to that of the "sustained" fast oscillations of wake or REM sleep episodes.

To characterize further this aspect, we investigated these brief periods of fast oscillations in more detail (Fig. 11). The correlation between neighboring sites showed that fast oscillations of SWS are characterized by correlations that fluctuate and stay high between neighboring sites for time windows of $\sim 100-500 \mathrm{msec}$ 

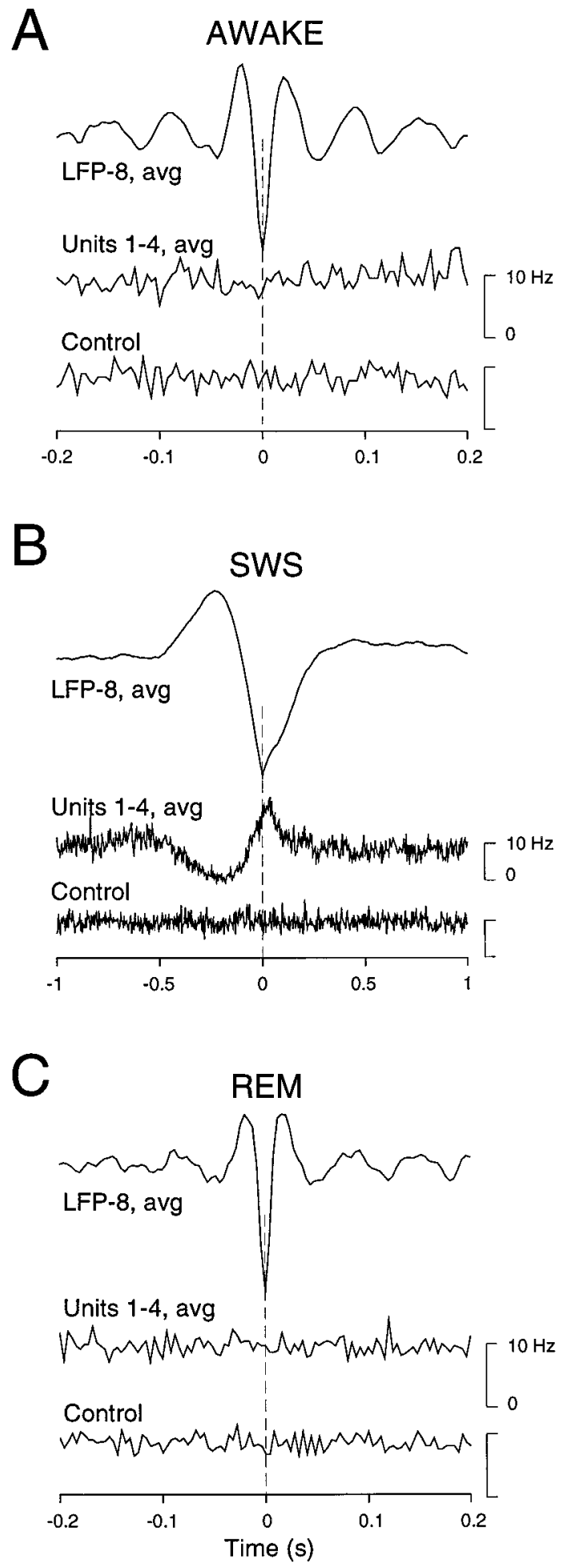

Figure 8. Correlations over large distances are present during slow-wave sleep but not during wake or REM sleep. Wave-triggered averages were computed from LFPs and from cells at a distance of $>4 \mathrm{~mm}$. LFP negativities from electrode $8(L F P-8, a v g)$ were detected to average units from electrodes 1 to 4 (Units 1-4, avg; the same procedures that were used in wave-triggered averages and the same data as in Fig. 6 for SWS and Fig. 7 for wake and REM sleep). $A$, In periods of wake, there was no visible relation between $L F P-8$ and units 1-4. B, During SWS in the same animal, the positivity/negativity complex was correlated with a decrease/increase of firing in units. $C$, No detectable relation was seen for REM sleep (same animal), similar to results in $A$. All control traces display the same procedure based on randomly shuffled spikes.
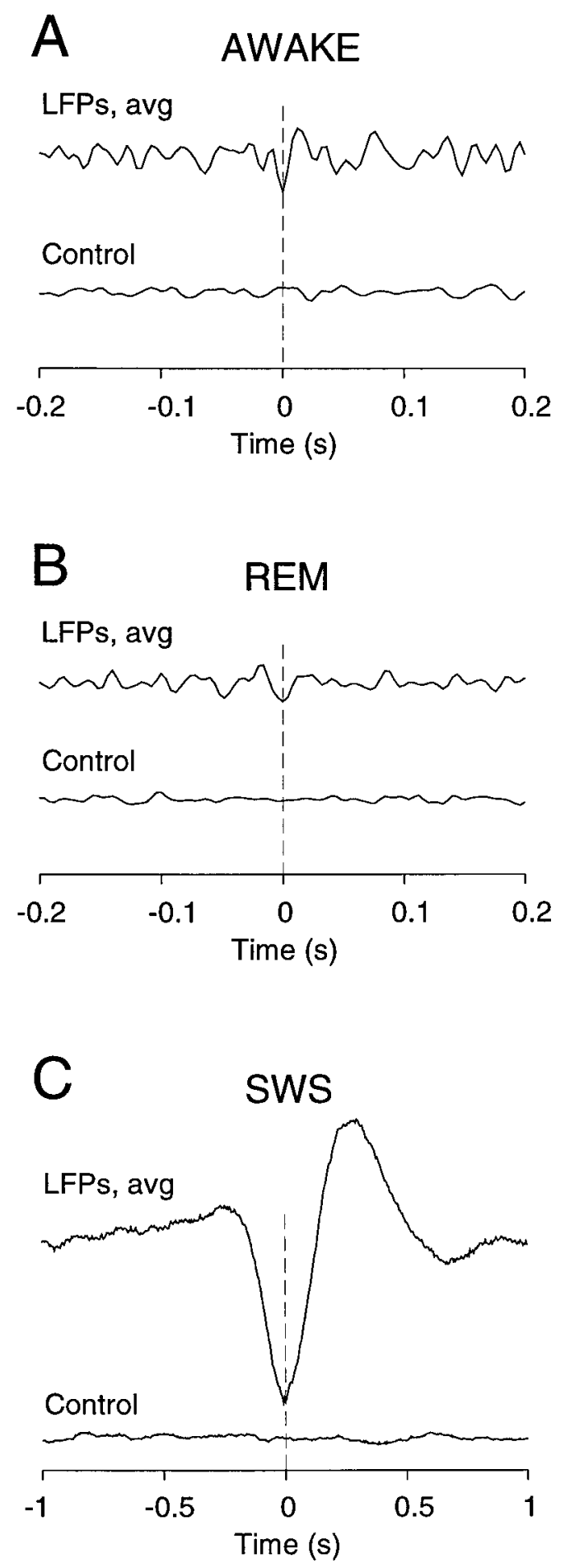

Figure 9. Relation between extracellular unit discharges and local field potentials as detected from spike events. Spike-triggered averages of local field potentials were first computed at each individual electrode. These spike-triggered averages were then averaged to yield a single curve, shown here for various states. $A$, In wake states, individual spikes were correlated with the negativity of the local field potentials ( $L F P s, a v g ; 5506$ spikes processed). $B$, A similar relation was obtained during REM sleep (LFPs, avg; 19491 spikes processed). $C$, During slow-wave sleep, the average LFP events corresponding to spikes consisted of a broad negative deflection, followed by a slow positive deflection (LFPs, avg; 34244 spikes processed; note different time scale). In all cases, the same analysis based on randomly shuffled spikes did not evidence any preferred pattern (Control). 
Figure 10. Fine structure of local field potentials during slowwave sleep. The LFPs at eight cortical sites (top curves; same experiment described in Fig. 1), the relative power of lowfrequency $(0.1-4 \mathrm{~Hz})$ and fast-frequency $(16-75 \mathrm{~Hz})$ components (middle curves), and the space constant of correlation decay with distance (bottom curve) are shown for a $20 \mathrm{sec}$ period of slow-wave sleep. Power spectra and spatial correlations were calculated in successive windows of $0.512 \mathrm{sec}(128$ points). Slow-wave complexes (single asterisk) were synchronous over the eight electrodes, whereas brief periods of fast oscillations (double asterisks) had lower spatial coherence.

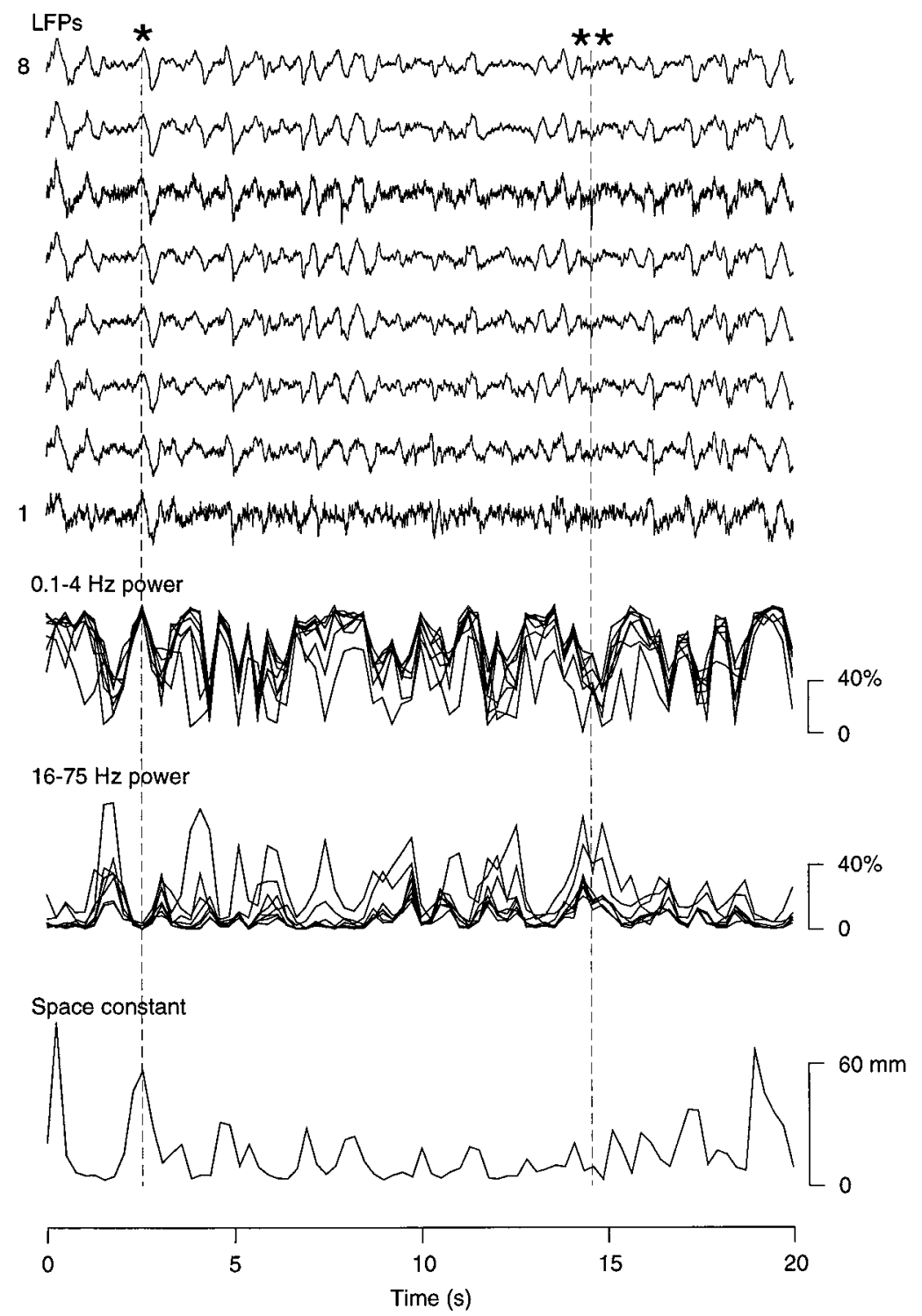

component correlated with an increased probability of firing in units adjacent to the recording electrode, but no relation was apparent with more distant units. Brief periods of fast oscillations with similar characteristics also appear during SWS.

\section{DISCUSSION}

In this paper, we have investigated the spatial and temporal distribution of fast and slow oscillation types occurring during natural wake and sleep states, as well as their relation with unit discharges. We summarize here the results obtained, relate them to previous approaches, and discuss possible mechanisms and physiological consequences of these findings.

\section{Spatial and temporal aspects of wake and sleep oscillations}

The findings of the present study can be summarized as follows: (1) slow-wave complexes of natural sleep are coherent across a distance of several millimeters in the cortex and are correlated with a concerted decreased and/or increased firing in units; (2) fast oscillations of wake and REM sleep are characterized by less global and more fluctuating coherence, and their depth-negative same pattern is seen coherently over large cortical distances $(>7$ $\mathrm{mm})$. By contrast, fast oscillations have their depth-negative LFP
Finally, the relation between field potentials and cellular events suggested by the present analysis is summarized in Figure 12. Slow-wave complexes are correlated with periods of decreased neuronal firing, followed by periods of increased firing, and the 


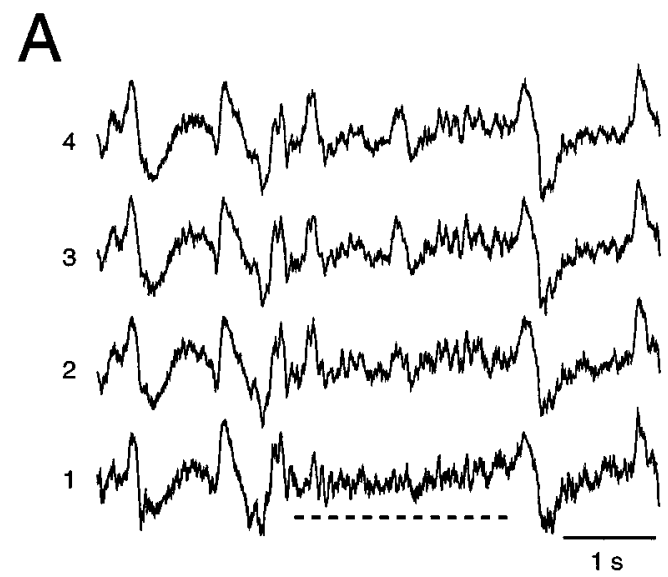

B

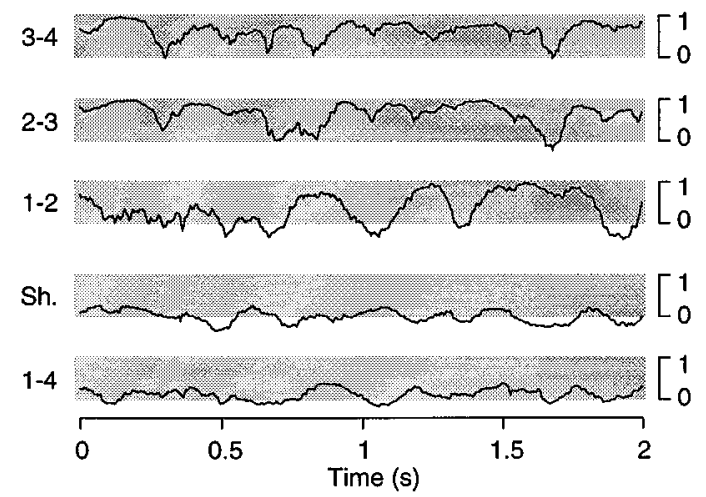

C

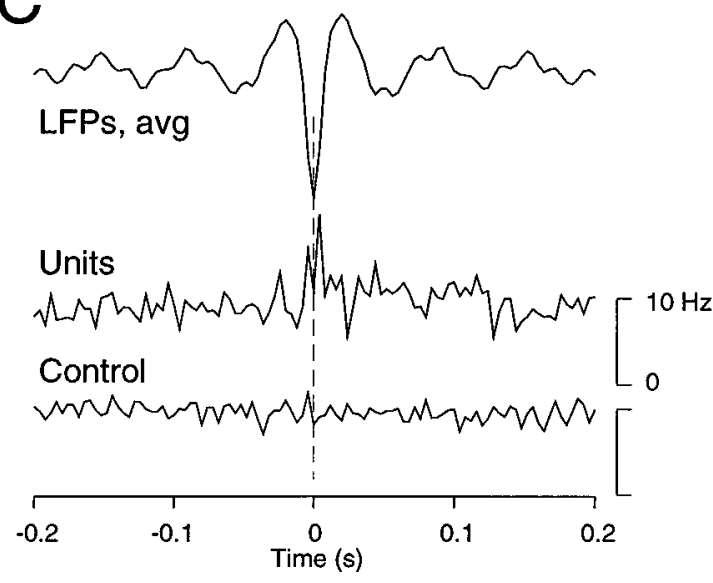

D

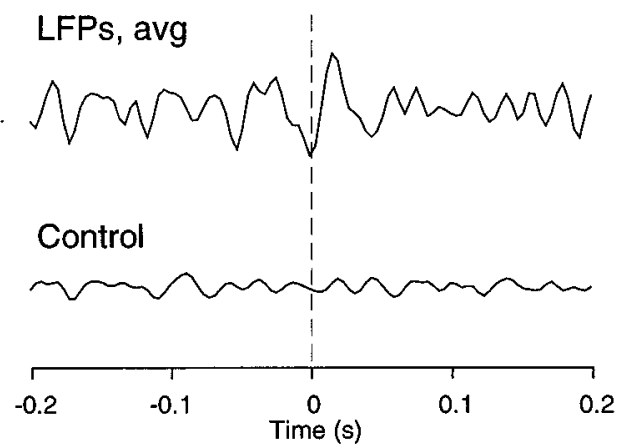

Figure 11. Fast oscillations during slow-wave sleep have characteristics similar to that during wake and REM sleep. $A$, A brief period of fast oscillations (dashed horizontal line) during slow-wave sleep. $B$, Dynamics of correlations during the period of fast oscillations shown in $A$, analyzed similarly as described in Figure 3. Fast oscillations displayed local patterns of synchrony, within short time windows and between neighboring electrodes, similar to that of wake and REM periods. $C$, Relation between neuronal firing and local field potentials. Slow-wave complexes were artificially removed from LFPs in a period of $11 \mathrm{~min}$ of slow-wave sleep. The corresponding spikes were also removed from multiunit discharges. The resulting LFPs and multiunit discharges were then analyzed similarly as described in Figure 7 (390 events processed). The wave-triggered averaging procedure shows that the negative LFP of fast oscillations of SWS (LFPs, avg) was correlated with an increase of firing (Units). The same analysis based on randomly shuffled spikes did not show any pattern (Control). D, Spike-triggered averages calculated similarly as described in Figure 9 (4011 spikes processed). Spikes were correlated with the LFP negativity of fast oscillations (LFPs, avg), similar to that of wake and REM sleep, but did not show any preferred pattern if spikes were randomly shuffled (Control).

EEG components are correlated with an increased probability of unit firing; and (3) SWS contains brief periods of fast oscillations whose spatiotemporal properties are similar to that of the sustained fast oscillations of activated states. We consider each of these points successively.

(1) We found that the slow-wave complexes of natural sleep display high spatial coherence. It was shown previously that low-frequency EEG components have higher coherence than do fast frequencies in rabbits and rats (Bullock and McClune, 1989) as well as in humans (Achermann and Borbély, 1998; but see Bullock et al., 1995). The present results are in agreement with this finding and further show that the spatial coherence of oscillations, as quantified by the correlation space constant, varies across wake and sleep states in parallel to classical criteria of LFP, EOG, and EMG changes (Fig. 5). This suggests that the global versus local level of coherence of LFPs is not only characteristic of the frequency band but also parallels the state of the animal.

However, it has been argued that the coherence estimated from LFPs or EEGs may reflect spurious correlations attributable to the filtering properties of extracellular space (Srinivasan et al., 1998). It may therefore be that the low-pass filtering nature of extracellular space (Nunez, 1981) induces artificial correlations selectively for low-frequency events. However, two arguments demonstrate that this is not the case here. First, fast oscillations may occasionally display high coherence similar to that of lowfrequency oscillations (Fig. 4A). Second, a consistent relation between LFP and cell firing extends to considerable cortical distances for slow-wave complexes but not for fast oscillations (Fig. 8).

We also found that slow-wave complexes display a striking correlation with unit firing; depth-LFP negativities are associated with increased firing, whereas depth-EEG positivities are simultaneous with a decreased firing, consistent with intracellular recordings during ketamine-xylazine anesthesia (Steriade et al., 1993a; Contreras and Steriade, 1995). These observations are also consistent with previous findings for delta waves in various preparations (Frost et al., 1966; Ball et al., 1977; Buzsáki et al., 1988). The same relations between the two main LFP components and 


\section{Slow-wave complexes}

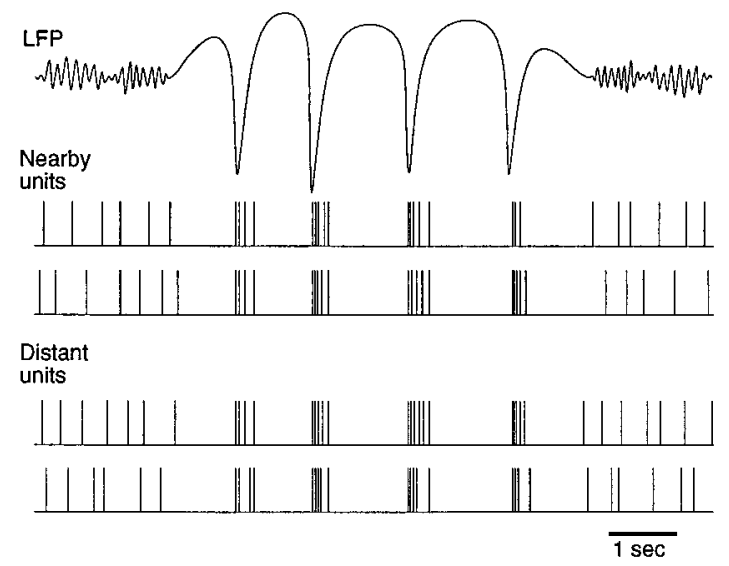

Fast oscillations

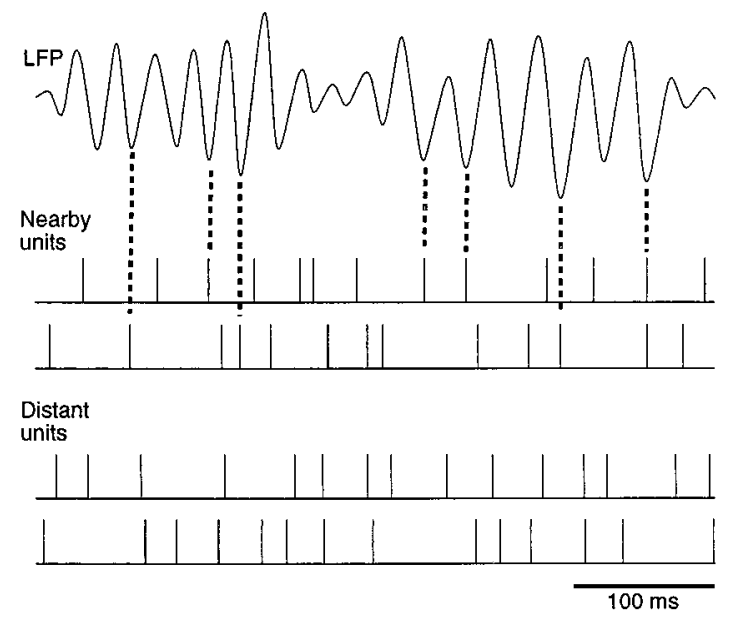

Figure 12. Schematic representation of the relationship between local field potentials and unit discharges during wake and sleep states. Top, During slow-wave sleep, slow-wave complexes were correlated with phasic-firing activity. Periods of neuronal silence coincided with depth positivity in the LFP, whereas depth-negative components occurred in coincidence with increased firing in units. The occurrence of periods of decreased and increased firing was synchronous over large cortical distances $(>7 \mathrm{~mm})$. Bottom, During fast oscillations, units discharged more tonically, with an increased probability of firing during the depth-negative component of the LFP. The coherence extended over short distances ( 1-2 mm), and unit activity was correlated only with the nearby LFP. This pattern was seen for fast oscillations of wake and REM sleep, as well as for brief periods of fast oscillations occurring during SWS.

sequences of depolarization and hyperpolarization were observed recently in intracellular recordings performed in awake and naturally sleeping cats (Steriade et al., 1999). In addition, the present analysis indicates that the periods of increased and decreased firing, occurring during slow-wave complexes, are coherent over several millimeters in the cortex (Fig. 6).

(2) During waking and REM sleep, we found here that the coherence in the $\beta-\gamma$ frequency band was highly fluctuating both in space and time, as neighboring electrodes typically synchronized for short periods of time (100-500 msec; see Figs. 3, 4). Similar spatiotemporal properties of local coherence were observed for fast oscillations in anesthetized animals, both for spontaneous oscillations (Steriade and Amzica, 1996) and for oscillations evoked by sensory stimuli (Eckhorn et al., 1988; Gray et al., 1989, 1992).

In wake and REM sleep periods, the discharge of units was related to the depth negativity of LFPs, similar to the fast oscillations occurring in anesthetized animals (Eckhorn et al., 1988; Gray and Singer, 1989; Murthy and Fetz, 1992; Steriade et al., 1996). However, we could not detect any significant correlation between LFP and units from distant electrodes (Fig. 8), in agreement with the local correlation displayed by LFPs. This is also in agreement with findings in anesthetized animals that evidenced that the correlation between distant cells was state dependent and was only present when the EEG displayed slow-wave activity (Contreras and Steriade, 1997).

(3) The fact that brief periods of fast oscillations are present between slow-wave complexes was shown previously in cat suprasylvian cortex during ketamine-xylazine anesthesia (Contreras and Steriade, 1995) and natural sleep (Steriade et al., 1996). We found here that the spatial and temporal coherence of these brief periods of fast oscillations, as well as their relation with unit discharges, is indistinguishable from that of the sustained fast oscillations of wake and REM sleep. However, the present analysis only investigated oscillations over distances up to $7 \mathrm{~mm}$ in the cerebral cortex, and further experiments are required to demonstrate that the above conclusions also apply to larger cortical distances.

\section{Mechanisms of coherent cortical oscillations}

The presence of a widespread decrease of firing suggests that slow-wave complexes of natural sleep are generated by a generalized disfacilitation in the cortex, followed by a rebound of the network, as found intracellularly during ketamine-xylazine anesthesia (Contreras and Steriade, 1995). The synchrony of the slow oscillation of ketamine-xylazine anesthesia is disrupted by the sectioning of intracortical connections (Amzica and Steriade, 1995) and is resistant to thalamic lesions (Steriade et al., 1993b), which suggested that it is generated intracortically. It is possible that similar mechanisms underlie the slow-wave complexes of natural sleep, although no precise biophysical mechanism has been proposed yet.

However, the intracortical synchrony is restored a few hours after the sectioning of intracortical connections (Amzica and Steriade, 1995). This may suggest that thalamocortical mechanisms may play an important role in establishing large-scale coherence. A recent computational model proposed a mechanism for large-scale synchrony based on thalamocortical loops (Destexhe et al., 1998a). It is possible that both intracortical and thalamocortical interactions play an important role in the largescale coherence of SWS. These possibilities should be addressed by future computational models.

Intracortical mechanisms were advanced to explain the synchrony of fast oscillations in the cerebral cortex on the basis of networks of inhibitory interneurons (Buzsáki and Chrobak, 1995; Traub et al., 1996). However, the question of how the same thalamocortical circuits are capable of generating low-frequency oscillations with large-scale coherence, as well as fast oscillations with local coherence, is still open. A model was proposed to explain these variations of coherence on the basis of twodimensional networks of excitatory and inhibitory neurons subject to pacemaker inputs of various frequencies (Destexhe and Babloyantz, 1991). Although this model displayed low-frequency 
oscillations with large coherence and fast oscillations with low coherence, it did not include the important role of corticothalamic feedback. More accurate models of thalamocortical circuits are needed to investigate mechanisms for the coexistence of oscillations of different levels of coherence.

\section{Possible physiological consequences}

The observation that highly coherent slow-wave patterns alternate with brief periods of low-coherent fast oscillations can be interpreted in three possible ways. First, it may be that fast oscillations constitute a background activity always present in thalamocortical networks and that this activity is regularly interrupted by slow-wave events. Slow-waves would therefore sculpt spatiotemporally coherent events into a background of lowcoherent fast oscillations.

A second possibility is that the fast oscillations occur in rebound to slow-wave events. Slow-waves are likely to be associated with a widespread hyperpolarization in cortical cells, followed by a rebound depolarization, as occurs in neocortical neurons during ketamine-xylazine anesthesia (Contreras and Steriade, 1995). At the cellular level, fast oscillations may occur following depolarization in neocortical cells attributable to intrinsic mechanisms (Llinás et al., 1991; Nuñez et al., 1992; Gutfreund et al., 1995; Gray and McCormick, 1996; Steriade et al., 1998). It is thus possible that short periods of fast oscillations appear during the rebound depolarization as a consequence of intrinsic ionic mechanisms in cortical cells.

A third possibility is that SWS iterates a cyclic process, in which brief periods of processing similar to that of the awake state alternate with highly synchronized network events. If the network rebounds synchronously after a period of silence, pyramidal cells should receive strong EPSPs followed by IPSPs, which form an ideal signal to trigger a massive calcium entry in the dendrites (Contreras et al., 1997). Because calcium is implicated in various forms of plasticity and long-term cellular changes (for review, see Ghosh and Greenberg, 1995), it is possible that slow-wave complexes are important to establish permanent changes in the network. The fast oscillations could reflect recalled events experienced previously, which are "imprinted" in the network via synchronized network events that appear as slow-wave complexes in the EEG.

\section{REFERENCES}

Achermann P, Borbély AA (1997) Low-frequency $(<1 \mathrm{~Hz})$ oscillations in the human sleep electroencephalogram. Neuroscience 81:213-222.

Achermann P, Borbély AA (1998) Coherence analysis of the human sleep electroencephalogram. Neuroscience 85:1195-1208.

Amzica F, Steriade M (1995) Disconnection of intracortical synaptic linkages disrupts synchronization of a slow oscillation. J Neurosci 15:4658-4677.

Amzica F, Steriade M (1997) The K-complex: its slow ( $<1 \mathrm{~Hz})$ rhythmicity and relation to delta waves. Neurology 49:952-959.

Ball CJ, Gloor P, Schaul N (1977) The cortical electromicrophysiology of pathological delta waves in the electroencephalogram of cats. Electroencephalogr Clin Neurophysiol 43:346-361.

Bouyer JJ, Montaron MF, Rougeul A (1981) Fast fronto-parietal rhythms during combined focused attentive behavior and immobility in the cat: cortical and thalamic localizations. Electroencephalogr Clin Neurophysiol 51:244-252.

Bullock TH, McClune MC (1989) Lateral coherence of the electrocorticogram: a new measure of brain synchrony. Electroencephalogr Clin Neurophysiol 73:479-498.

Bullock TH, McClune MC, Achimowicz JZ, Iragui-Madoz VJ, Duckrow
RB, Spencer SS (1995) Temporal fluctuations in coherence of brain waves. Proc Natl Acad Sci USA 92:11568-11572.

Buzsáki G, Chrobak JJ (1995) Temporal structure in spatially organized neuronal ensembles: a role for interneuronal networks. Curr Opin Neurobiol 5:504-510.

Buzsáki G, Bickford RG, Ponomareff G, Thal LJ, Mandel R, Gage FH (1988) Nucleus basalis and thalamic control of neocortical activity in the freely moving rat. J Neurosci 8:4007-4026.

Contreras D, Steriade M (1995) Cellular bases of EEG slow rhythms: a study of dynamic corticothalamic relationships. J Neurosci 15:604-622.

Contreras D, Steriade M (1997) State-dependent fluctuations of lowfrequency rhythms in corticothalamic networks. Neuroscience $76: 25-38$

Contreras D, Destexhe A, Steriade M (1997) Intracellular and computational characterization of the intracortical inhibitory control of synchronized thalamic inputs in vivo. J Neurophysiol 78:335-350.

Destexhe A, Babloyantz A (1991) Pacemaker-induced coherence in cortical networks. Neural Comput 3:145-154.

Destexhe A, Contreras D, Steriade M (1998a) Mechanisms underlying the synchronizing action of corticothalamic feedback through inhibition of thalamic relay cells. J Neurophysiol 79:999-1016.

Destexhe A, Contreras D, Steriade M (1998b) Spatial and temporal relations between extracellular neuronal discharges and local field potential oscillations in cat cerebral cortex during natural wake and sleep states. Soc Neurosci Abstr 24:13.

Eckhorn R, Bauer R, Jordan W, Brosch M, Kruse W, Munk M, Reitboek HJ (1988) Coherent oscillations: a mechanism of feature linking in the visual cortex? Multiple electrode and correlation analyses in the cat. Biol Cybern 60:121-130.

Evarts EV (1964) Temporal patterns of discharge of pyramidal tract neurons during sleep and waking in the monkey. J Neurophysiol 27:152-171.

Freeman WJ, Van Dijk BW (1987) Spatial patterns of visual cortical fast EEG during conditioned reflex in a rhesus monkey. Electroencephalogr Clin Neurophysiol 422:267-276.

Frost JD, Kellaway PR, Gol A (1966) Single-unit discharges in isolated cerebral cortex. Exp Neurol 14:305-316.

Ghosh A, Greenberg ME (1995) Calcium signaling in neurons: molecular mechanisms and cellular consequences. Science 268:239-247.

Gray CM, McCormick DA (1996) Chattering cells: superficial pyramidal neurons contributing to the generation of synchronous oscillations in the visual cortex. Science 274:109-113.

Gray CM, Singer W (1989) Stimulus-specific neuronal oscillations in orientation columns of cat visual cortex. Proc Natl Acad Sci USA 86:1698-1702.

Gray CM, Konig P, Engel AK, Singer W (1989) Oscillatory responses in cat visual cortex exhibit inter-columnar synchronization which reflects global stimulus properties. Nature 338:334-337.

Gray CM, Engel AK, Konig P, Singer W (1992) Synchronization of oscillatory neuronal responses in cat striate cortex: temporal properties. Vis Neurosci 8:337-347.

Gutfreund Y, Yarom Y, Segev I (1995) Subthreshold oscillations and resonant frequency in guinea-pig cortical neurons-physiology and modeling. J Physiol (Lond) 483:621-640.

Hubel D (1959) Single-unit activity in striate cortex of unrestrained cats. J Physiol (Lond) 147:226-238.

Llinás RR, Ribary U (1993) Coherent $40 \mathrm{~Hz}$ oscillation characterizes dream state in humans. Proc Natl Acad Sci USA 90:2078-2081.

Llinás RR, Grace AR, Yarom Y (1991) In vitro neurons in mammalian cortical layer 4 exhibit intrinsic oscillatory activity in the 10 to $50 \mathrm{~Hz}$ frequency range. Proc Natl Acad Sci USA 88:897-901.

Lopes da Silva FH, van Rotterdam A, Storm van Leeuwen W, Tielen AM (1970) Dynamic characteristics of visual evoked potentials in the dog. II. Beta frequency selectivity in evoked potentials and background activity. Electroencephalogr Clin Neurophysiol 29:260-268.

Murthy VN, Fetz EE (1992) Coherent 25 to $35 \mathrm{~Hz}$ oscillations in the sensorimotor cortex of awake behaving monkeys. Proc Natl Acad Sci USA 89:5670-5674.

Nuñez A, Amzica F, Steriade M (1992) Voltage-dependent fast (20-40 $\mathrm{Hz}$ ) oscillations in long-axoned neocortical neurons. Neuroscience 51:7-10.

Nunez PL (1981) Electric fields of the brain. The neurophysics of EEG. Oxford: Oxford UP.

Press WH, Flannery BP, Teukolsky SA, Vetterling WT (1986) Numer- 
ical recipes. The art of scientific computing. Cambridge, MA: Cambridge UP.

Ribary U, Ioannides AA, Singh KD, Hasson R, Bolton JP, Lado F, Mogilner A, Llinás RR (1991) Magnetic field tomography of coherent thalamocortical $40 \mathrm{~Hz}$ oscillations in humans. Proc Natl Acad Sci USA 88:11037-11041.

Rougeul A, Bouyer JJ, Dedet L, Debray O (1979) Fast somato-parietal rhythms during combined focal attention and immobility in baboon and squirrel monkey. Electroencephalogr Clin Neurophysiol 33:23-59.

Srinivasan R, Nunez PL, Silberstein RB (1998) Spatial filtering and neocortical dynamics: estimates of EEG coherence. IEEE Trans Biomed Eng 45:814-826.

Steriade M, Amzica F (1996) Intracortical and corticothalamic coherency of fast spontaneous oscillations. Proc Natl Acad Sci USA 93:2533-2538.

Steriade M, Deschênes M, Oakson G (1974) Inhibitory processes and interneuronal apparatus in motor cortex during sleep and waking. I. Background firing and responsiveness of pyramidal tract neurons and interneurons. J Neurophysiol 37:1065-1092.

Steriade M, Nuñez A, Amzica F (1993a) A novel slow $(<1 \mathrm{~Hz})$ oscilla- tion of neocortical neurons in vivo: depolarizing and hyperpolarizing components. J Neurosci 13:3252-3265.

Steriade M, Nuñez A, Amzica F (1993b) Intracellular analysis of relations between the slow $(<1 \mathrm{~Hz})$ neocortical oscillation and other sleep rhythms of the electroencephalogram. J Neurosci 13:3266-3283.

Steriade M, Contreras D, Amzica F (1994) Synchronized sleep oscillations and their paroxysmal developments. Trends Neurosci 17:199-208.

Steriade M, Amzica F, Contreras D (1996) Synchronization of fast $(30-40 \mathrm{~Hz})$ spontaneous cortical rhythms during brain arousal. J Neurosci 16:392-417.

Steriade M, Timofeev I, Durmüller N, Grenier F (1998) Dynamic properties of corticothalamic neurons and local cortical interneurons generating fast rhythmic $(30-40 \mathrm{~Hz})$ spike bursts. J Neurophysiol 79:483-490.

Steriade M, Timofeev I, Grenier F (1999) Intracellular activity of various neocortical cell-classes during the natural wake-sleep cycle. Soc Neurosci Abstr, in press.

Traub RD, Whittington MA, Stanford IM, Jefferys JGR (1996) A mechanism for generation of long-range synchronous fast oscillations in the cortex. Nature 383:621-624. 